\title{
The influence of physical activity on mental well-being
}

\author{
Dr Kenneth R Fox* \\ Department of Exercise and Health Sciences, University of Bristol, Priory House, Woodlands Rd, Bristol, UK
}

Accepted: 7 May 1999

\begin{abstract}
Objective: The case for exercise and health has primarily been made on its impact on diseases such coronary heart disease, obesity and diabetes. However, there is a very high cost attributed to mental disorders and illness and in the last 15 years there has been increasing research into the role of exercise a) in the treatment of mental health, and b) in improving mental well-being in the general population. There are now several hundred studies and over 30 narrative or meta-analytic reviews of research in this field. These have summarised the potential for exercise as a therapy for clinical or subclinical depression or anxiety, and the use of physical activity as a means of upgrading life quality through enhanced self-esteem, improved mood states, reduced state and trait anxiety, resilience to stress, or improved sleep. The purpose of this paper is to a) provide an updated view of this literature within the context of public health promotion and b) investigate evidence for physical activity and dietary interactions affecting mental well-being.

Design: Narrative review and summary.

Conclusions: Sufficient evidence now exists for the effectiveness of exercise in the treatment of clinical depression. Additionally, exercise has a moderate reducing effect on state and trait anxiety and can improve physical self-perceptions and in some cases global self-esteem. Also there is now good evidence that aerobic and resistance exercise enhances mood states, and weaker evidence that exercise can improve cognitive function (primarily assessed by reaction time) in older adults. Conversely, there is little evidence to suggest that exercise addiction is identifiable in no more than a very small percentage of exercisers. Together, this body of research suggests that moderate regular exercise should be considered as a viable means of treating depression and anxiety and improving mental well-being in the general public.
\end{abstract}

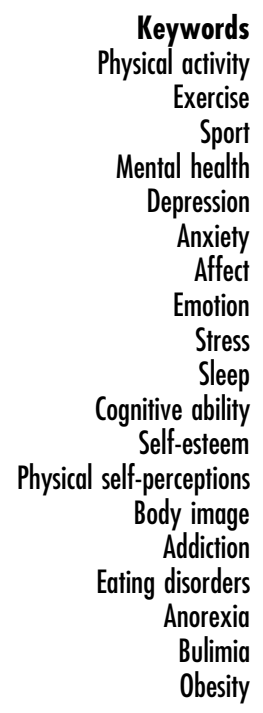

\section{Key messages}

- There is growing evidence demonstrating that exercise can be effective in improving the mental well-being of the general public, largely through improved mood and self-perceptions.

- There is good evidence to demonstrate that exercise is effective as a treatment for clinical depression and anxiety.

- Together this adds to the already convincing literature that exercise reduces morbidity and mortality from coronary heart disease, diabetes, obesity and some cancers.

- The interactive effect of diet and exercise and mental well-being has not been systematically studied.

\section{Introduction}

The case for exercise and physical health is now widely accepted by medical authorities across the world $^{1,2}$. Sedentary living doubles the risk of morbidity and mortality from coronary heart disease and stroke $e^{3,4}$ which is comparable with the risk associated with hypertension and hyperlipidaemia and not far behind that of smoking. In addition, low activity levels are thought to be a major cause of obesity ${ }^{5}$ and contributor to the rising incidence of diabetes and some cancers. The incidence of inactivity is also high in many developed countries where technology is slowly removing exercise from lifestyles and is estimated at around $40 \%$ of the middle aged and elderly in the $\mathrm{UK}^{6}$. The public health burden of inactivity is therefore problematic and expensive ${ }^{7}$ and activity promotion could provide a cost-effective strategy for improvement ${ }^{8}$.

While physical activity can indirectly improve subjective well-being and life quality by keeping disease and premature death at bay, there has recently been an increasing interest in its direct role in the prevention and treatment of mental health problems. Mental illness and disorders are widespread and possibly on the increase. The 1995 Health Survey for England ${ }^{9}$, for example, showed that $20 \%$ of women 
and $14 \%$ of men have at some time suffered mental health problems. Up to $20 \%$ of children will suffer mild and $7-10 \%$ moderate to severe mental health problems that hinder normal development ${ }^{10}$ and there is evidence of a worsening trend, particularly in socially disadvantaged populations ${ }^{11}$. Depression is the most widespread disorder affecting $5-10 \%$ of the populations of most developed countries ${ }^{12}$ with $20 \%$ of those presenting in primary care in Britain having recognizable degrees of symptomology ${ }^{13}$. The cost is therefore high with the British Department of Health ${ }^{14}$ estimating $17 \%$ of expenditure in health services spent on mental illness and disorders through hospitalization, drug therapy and consultations. Other costs are incurred through social services provision, sickness and invalidity benefits, and loss of work productivity, with $15 \%$ and $26 \%$ of days of certified incapacity in men and women respectively being caused by mental disorders.

Human suffering is high. Mental illness is socially debilitating and associated with suicide ideation and attempts, drug and alcohol abuse and homelessness. For every serious case, however, there are also many more individuals who suffer a general malaise of low mental well-being characterized by emotional distress, low self-esteem, poor body image, sense of hopelessness, chronic stress and anxiety. This is often not clinically diagnosed and so its incidence is difficult to accurately assess but it has major implications for other problems such as heavy drinking, smoking, absenteeism from work, family breakdown, physical violence and abuse, and quality of life. These problems seem to be present in westernized countries regardless of the stability of economic or political climate.

Physical activity can be viewed from four different perspectives regarding its direct contribution to solving mental health problems:

1 Treatment of mental illness and disorders;

2 Prevention of mental illness and disorders;

3 Improvement of mental and physical well-being of those with mental illness;

4 Improvement of mental well-being of the general population.

The study of exercise and mental health is not new. The "feeling good", "warm glow", or "exercise high" phenomenon that is widely reported anecdotally, has received increasing research attention in the past decade. In 1987 the US National Institute of Mental Health consensus workshop statements regarding the contribution of exercise to mental health were published ${ }^{15}$. Since that time there have been several books dedicated to the topic ${ }^{16,17,18}$ along with scores of published narrative and meta-analytical reviews. Recently, a literature review was commissioned and a national consensus conference held in Britain by Somerset Health Authority. In Belgium ${ }^{19}$, psychomotor therapy to treat depression and anxiety is already offered through the state health provision. The literature focuses on the impact of physical activity on depression, anxiety and stress, emotion, mood and well-being, self-esteem and self-perceptions, sleep quality, and the negative effects of exercise.

\section{The effects of physical activity on mental health}

\section{Depression}

Mild depression is characterized by a period of frequent episodes of unhappiness. It is relatively common and often not diagnosed. However, clinical depression is determined against diagnostic criteria through questionnaires such as the Beck Depression Inventory ${ }^{20}$ or interviews such as DSM-III ${ }^{21}$ or Research Diagnostic Criteria $^{22}$. There is evidence from four prospective epidemiological studies that those who become or remain active or fit are less likely to suffer clinical depression. Odds ratio over a period of 9 years for those who remained low in activity was 1.22 and for those became inactive was 1.61 against a baseline of high activity on both occasions ${ }^{23}$. Paffenbarger, Lee, and Leung ${ }^{24}$ recorded a dose response relationship for men over a $23-27$ year period, with those engaged in high activity (>2500 kcals/week) at 28\% reduced risk, and moderate activity (1000-2499 kcals/week) at 17\% risk for becoming depressed compared to those low in activity. Farmer et al. ${ }^{25}$ found twice the risk of clinical depression in women low in exercise over an 8-year period.

Caution needs to be applied to such studies where both variables provide plausible causal explanations of the relationship between them, however the existence of a dose response for exercise on depression reduction over time is convincing.

Two recent reviews have focused on the effect of exercise on clinical depression. Mutrie ${ }^{26}$ summarized 10 key randomised controlled studies. Craft and Landers ${ }^{27}$ conducted a meta-analysis on all available studies where an exercise therapy for clinical depression had been used and produced a large mean effect size of -0.72 . Mutrie concluded:

- Physical activity is associated with decreased risk of developing clinical depression.

- Experimental studies show that aerobic and resistance exercise are effective in treating depression.

- The effect is of the same magnitude as psychotherapeutic interventions.

This is strong evidence that exercise can help prevent and treat this common cause of mental illness and threat to mental well-being. 


\section{Anxiety and stress reactivity}

Several narrative and meta-analytic reviews have been conducted in this area such as Calfas and Taylor $^{28}$ with adolescents, McDonald and Hodgdon ${ }^{29}$, Petruzzello et al. ${ }^{30}$ and Taylor ${ }^{31}$. Research has taken three approaches. First, the effect of a single bout of exercise on state anxiety (acute or right now feelings) as been addressed. This literature has indicated moderate effects for reductions in anxiety post exercise with most studies testing the effects of aerobic forms of exercise such as running. Second, the effects of engaging in several weeks of an exercise programme on both state and trait anxiety (predisposition to react nervously) have been investigated. Similarly, exercise has been shown to provide moderate reductions in state and trait anxiety. Third, the effect of single exercise sessions and exercise programmes has been tested on the psychosocial and psycho-physiological reactivity to a subsequent psychological stressor such as a complex mental task or public speaking. This literature has provided equivocal results with only half the studies showing a benefit from exercise training or improved fitness although this may be as much due to the difficulties associated with measurement as lack of effect.

\section{Subjective well-being, emotion and mood}

The ability of physical activity to "energise" and produce more positive mood is reported widely. At least 20 reviews of studies in the area of affect have been published. Stephens ${ }^{32}$ summarised epidemiological evidence that showed a clear association between activity and several indices of subjective well-being. Biddle ${ }^{33}$ has recently conducted a review of reviews on the literature and concluded the following.

- Large scale surveys in several countries using different methods and criteria confirm a moderate association between physical activity and indices of subjective well-being.

- Experimental studies support a positive effect on mood for moderate intensity exercise.

- Affective benefits are more likely to be experienced if participants focus on personal improvement goals.

Both survey and experimental research therefore provide support to the well publicised statement that "exercise makes you feel good".

\section{Self-esteem and self-perceptions}

Self-esteem is important when studying mental wellbeing because of (a) its close association with emotional stability and adjustment, (b) low self-esteem features in many forms of mental illness and (c) low self-esteem is associated with poor health behaviours. It is therefore regarded as both an indicator of well-being and as a marker for recovery. Some psychologists would go so far as to say that self-esteem is the core of mental health as it represents our self-rating of overall worth.

Fox $^{34}$ and Spence and Poon ${ }^{35}$ recently reviewed studies to establish the impact of exercise on self-esteem. Both reviews concluded that there is an inconsistent and weak relationship between physical activity and global self-esteem. The meta analysis of Spence revealed a weak mean effect size of 0.22 . Fox identified 36 randomized controlled studies and 44 controlled studies since 1971 and found that approximately 50\% indicated positive changes in self-esteem. However, this finding is in line with theoretical reasoning that would suggest that self-esteem is a stable construct and not easily changed by success in a single life domain. Self-esteem theory and measurement has advanced considerably in the last decade and more recent studies are focusing on the physical self ${ }^{36}$ which consists of self-ratings of the body, its appearance, its capabilities and worth. Physical self-worth has been shown through its associations with measures of emotional adjustment, independent of global self-esteem and socially desirable responding, to have mental wellbeing properties in its own right ${ }^{37}$. There is clear evidence that exercise can change people's perceptions of their physical self and identity in a positive way. For some people, in some circumstances, particularly those who are initially low in self-esteem, this extends to more generalized changes in self.

\section{Cognitive performance}

There has been some interest in the role of exercise in improving mental agility, particularly in older people, the view being that declines might be preventable. Boutcher ${ }^{38}$ and Etnier et al. ${ }^{39}$ recently reviewed the impact of exercise on cognitive function (reaction time, memory, and fluid intelligence) in older people. Boutcher concluded that though cross sectional studies show that fit older adults display better cognitive functioning than unfit older adults, the evidence from experimental studies remains equivocal with 5 intervention studies demonstrating improvements but 9 not showing significant change. Etnier's et al. meta analysis which focused on the relationship between fitness and cognitive performance in older adults showed a weak but significant overall effect size. It appears that the view that exercise into old age keeps you alert and spritely has yet to be substantiated fully. There are insufficient numbers of well-conducted studies in younger adults and children to draw conclusions.

\section{Sleep quality}

Insomnia affects approximately a third of the adult population and is associated with poor work performance 
and psychological dysfunction ${ }^{40}$. The interest in the effect of exercise has grown with the acceptance that sleeping pills have undesirable side effects including increased risk of mortality and dependence. Epidemiological evidence demonstrates that daylight exercise is the behaviour most closely related to sleep quality. Youngstedt ${ }^{41}$ recently produced a meta analysis of 38 studies on the effects of a single bout of exercise on subsequent sleep of good sleepers. A number of conclusions were drawn:

- Exercise had no effect on the time it took to fall asleep.

- Exercise produced small increases in the amount of sleep time and slow-wave sleep.

- Exercise reduced rapid eye movements (REM) and increased the time before onset.

The effect of exercise programmes on the sleep quality of poor sleepers was assessed in two well designed randomized controlled studies and exercise has been shown to have a moderate positive effect although as yet it is not clear if exposure to bright light through exercise is a contributing mechanism. This appears to be a fruitful area of research but current evidence would suggest that exercise in bright light, with emphasis on duration rather than intensity, regardless of fitness level will improve sleep, regardless of usual sleep quality.

\section{Negative effects of physical activity}

There have been frequent efforts in the media to find negative aspects of exercise and the concept of exercise addiction is often targeted. Szabo ${ }^{42}$ recently addressed the literature to examine the existence of negative effects. He concluded that exercise dependence exists as a psychopathological disorder but is extremely rare. He suggested that exercise dependence is often confused with a high degree of exercise commitment and outlined clear distinctions between the two based on positive versus negative motivation and the degree of centrality of exercise in life. He cautioned that there are a significant number of people with eating disorders who use exercise to promote weight loss but these can be distinguished from highly committed exercisers by their motivational profile. Female athletes, particularly those in sports where slenderness is required for performance and aesthetics, are at higher risk for developing eating disorders ${ }^{43}$. Similarly those who seek high level athletic performance and/or body building are probably more likely to take banned substances ${ }^{42}$.

\section{Summary}

Evidence is convincing that exercise can be useful in treating and avoiding depressive illnesses, and can be used as a means of reducing stress and anxiety on a daily basis. In addition, there is sufficient evidence to show that single bouts of activity can improve mood and sleep quality and that people who are more active are much more likely to rate themselves and their sense of mental well-being more positively. The feeling good effect of exercise therefore seems to be substantiated by research. In addition, regular exercise appears to offer a vehicle for more deep-seated change through improvements in the way we view our physical selves, and this has potential to generalize to higher self-esteem and identity change. Any negative mental effects from exercise seem to affect only a small percentage of participants.

\section{Mechanisms for mental bealth improvement}

Although there has been considerable progress in the past decade on establishing the effect of exercise on elements of mental health and well-being, the specific mechanisms and the conditions under which they operate have not yet been determined. Several candidates have been identified and to varying degrees these are undergoing research. Mechanisms fall under three categories; biochemical, physiological, and psycho-social.

\section{Biochemical mechanisms}

Considerable media attention has arisen from the discovery of endogenous opioid peptides in blood during and after exercise. The view was that these substances, primarily plasma $\beta$-endorphin, produced a feeling of euphoria or "runners high". Subsequent analysis has not supported this as a widespread effect as few exercisers report these sensations. It is likely to require high levels of exercise output, and further studies have not found a correlation between endorphin levels and mood. Measurement, control and isolation difficulties make this a challenging area of research and there has been little systematic progress since the early $1990 \mathrm{~s}^{44}$. Dishman ${ }^{45}$ recently summarized the evidence for norepinephrine as a trigger for anxiety and depression reduction and reported similar difficulties.

There has been recent attention paid to the interaction between physical activity and central serotonin (5-hydoxytryptamine [5-HT]) as a mood enhancer ${ }^{46}$. Animal and human studies have supported increases in brain 5-HT synthesis and metabolism with acute exercise but the extent to which this is a trigger for improved mood remains unknown.

\section{Physiological mechanisms}

There is a close relationship in adults between participation in aerobic activity and cardiovascular 
fitness. Therefore, epidemiological studies that have used fitness or activity measures have often produced similar relationships with measures such as subjective well-being. However, in experimental studies, physical fitness change rarely produces more than a weak association with either reduction in depression ${ }^{26,27}$ and anxiety or increases in mood and self-perceptions ${ }^{34,35}$. It appears from these data that increased fitness may not be the trigger for change although it may eventually accompany it. Process factors associated with regular participation in exercise rather than change in functional status seem to be more salient to mental well-being change.

Thermogenesis or increased body core temperature through acute exercise has also been suggested as a trigger of increased relaxation and improved mood. To date, findings have been inconsistent ${ }^{47}$. Other suggested mechanisms that have received some support have included increased muscle relaxation, cerebral blood flow and neurotransmitter efficiency.

\section{Psychosocial mechanisms}

Four areas are being investigated. Improvements in perceptions of competence and self-efficacy or confidence about the body and its capabilities may be increased through regular exercise and this may generalize to global self-esteem and other elements of well-being. This may be particularly important for older people or individuals recovering from illnesses and injury ${ }^{48}$.

Body image is more closely related to self-esteem than any other single element of self, particularly for females ${ }^{36,43,49}$. It is often so central that it influences daily habits, patterns of food choice, choice in clothes, confidence in public, and for many carries frequent emotional signals that can influence mood. Physical activity offers a means of improving body image through fat loss and improved muscle fitness. However, there is a complex of constructs involved including body satisfaction and acceptance and some studies indicate (often with females high in obsessiveness and neuroticism) that exercise raises body awareness and expectations and is counterproductive to satisfaction and self-esteem ${ }^{43}$.

One of the potentially fruitful areas of research is the contribution of mastery and self-determination to self-esteem and mental well-being. Exercise can provide a medium for slowly taking command over health behaviour and body appearance that may trigger a general sense of autonomy. The important effect may not so much be perceived change but the empowerment that achieving the change provides ${ }^{36}$.

Finally there has been less of a focus on the social benefits of exercise than is warranted. Social affiliation and significance are related to self-esteem ${ }^{49}$ and social apathy is an established corollary of depression. The social interaction that activity can provide through sport participation and joining exercise groups may provide social support for improving self-esteem and life satisfaction. In older people, activity may increase mobility and independence and promote social interaction.

In summary, the mechanisms underpinning improved well-being and alleviation of distressing mental illness have been difficult to isolate and establish. It is likely that multiple mechanisms are effective in any one situation. The dominance of any one mechanism will be determined by exercise characteristics such as intensity and duration, characteristics of the individual, and environmental factors surrounding the exercise. Currently the evidence suggests that factors associated with the process of exercise rather than the physiological adaptations resulting from regular exercise training are primarily responsible for improvements in short and long-term well-being.

\section{Activity and dietary interactions}

There is no body of knowledge specifically directed at the interaction between diet and exercise in mental health. In the latest book summarizing research in physical activity and mental health ${ }^{18}$ diet and nutrition are not listed in the index. There is a general absence of systematic research into the conjoint effects of nutrition, dieting and exercise and sport participation and this applies to issues in sport and exercise performance, as well as physical and mental health outcomes. This suggests that joint forums are required to generate new ideas, hypotheses, and research collaboration between the two academic bodies.

There are some key areas where interactive effects are being researched or requiring multidisciplinary attention.

\section{Exercise and anorexia}

Starvation and excessive exercising coexist in anorexics who are obsessive-compulsive and/or who have a high degree of neuroticism. Recent animal studies have indicated that when deprived of food and given the opportunity to exercise on a running wheel they will increase physical activity ${ }^{50}$. This would intuitively be the opposite behaviour expected for self-preservation. Although in the early stages of research, a mechanism has been hypothesized which involves the interactive effects of starvation and increased exercise on 5-HT (serotonin $)^{51}$.

\section{Exercise and food intake}

Although not directly related to mental health but implicated through its effect on obesity development and weight management attempts, are the interactive effects of energy intake and energy expended through exercise. Recently, Blundell and King ${ }^{52}$ addressed several 
key issues in this area and concluded: Exercise produces a reduction in appetite. However this is relatively short lived and lasts in most cases less than an hour.

There is no evidence of tight coupling between energy deficit created through exercise and subsequent energy intake. This suggests that exercise for weight loss is not futile as there is not a strong physiological link to stimulate compensatory eating.

There is stronger regulation of carbohydrate and protein than fat in response to exercise. This suggests that hunger following exercise is more likely to be driven by need for carbohydrate repletion in the muscles and protein for synthesis. Post exercise meals low in fat would therefore promote successful weight management.

Clearly this is an important area of research as mental health is often influenced by guilt, low self-efficacy or futility regarding maintenance of a lean body.

\section{Exercise and sustained weight loss}

There have been a series of studies from different research groups showing that those who maintain exercise following a weight loss programme, are much more likely to sustain their weight loss. This cannot be fully explained by the increased energy expended by the exercise. The view exists therefore that psychological mechanisms such as increased selfesteem, confidence and control developed through the exercise is promoting more successful management of energy intake ${ }^{53}$. The relative influence of exercise and eating behaviour and their interactive effects on long term weight management success is an important issue as it affects large numbers of individuals.

\section{Other issues}

Other areas of dietary/exercise interaction of potential importance include:

- The interactive effect of low food intake, low blood sugar and high activity on mood and mental and physical performance at school and work.

- The interaction between macronutrient intake and exercise intensity and duration on acute and chronic mental well-being indicators.

\section{Issues and recommendations}

\section{Research issues}

Physical activity and mental health remains an underresearched and under-invested area compared to other fields of health service research. For example, the work on exercise and self-esteem amounts to little more than one randomized controlled study per year for the last 30 years.
Most studies have been conducted by academics in the field of exercise, sport and health science and evidence-based-health principles have not been adopted widely. For example, intention to treat statistics are rarely included in intervention studies. There is also little evidence that cost effectiveness has been considered, and criteria for randomized controlled studies have only rarely been fully satisfied.

As with most behavioural studies, findings are limited to consenting volunteers who can tolerate the exercise session and/or who adhere to the exercise programme. Whereas this may be acceptable for the design of a health service intervention, more research is needed on those who dropout or avoid programmes so that future work might address mediating factors which prevent mental health benefits.

The area suffers from a "so what" syndrome. It is not often clear what the practical and clinical significance of improvements in some constructs such as subjective well-being, self-perceptions, and mood actually are. This is particularly the case where well-being of the general public who are not mentally ill is being addressed. Studies are required that investigate change alongside reduction in GP visits, reliance on medication and other indicators of well-being such as emotional adjustment, social functioning and life satisfaction.

This article has clearly outlined the scope for collaborative research between nutritionists and exercise scientists to investigate interactive effects of food intake and activity on mental health.

\section{Exercise and bealth promotion}

Exercise should be promoted regardless of its impact on mental health as it carries significant reduction in risks for a range of diseases and disorders for all sectors of society. The body of literature summarized in this article also supports the need for exercise promotion in the general public as it could prove to be a cheap and available vehicle for improving self-perceptions, mood, life satisfaction, social interaction and quality of life. However, there is also sufficient evidence to support the use of exercise in the treatment of depression, anxiety and stress. Treatment interventions should be tried and tested for cost effectiveness in field settings.

There is no definitive exercise recommendation for all elements of mental health promotion as it is likely that different formulas of frequency, intensity and duration of exercise apply for different mechanisms and perhaps different populations. However, the current general message of 30 minutes or more of moderate activity such as brisk walking on 5 or 6 days each week is supported by the literature. Longer may be better, resistance exercise should also be considered and certainly for those already fit and active, 
participation in vigorous exercise and sport may well bring further benefits in mental well-being.

\section{Acknowledgements}

This overview has drawn upon recent work commissioned by Somerset Health Authority, UK, directed by Trudy Grant of the Somerset Physical Activity Group. Thanks go to colleagues Stuart Biddle, Steven Boutcher, Nanette Mutrie, Attila Szabo, and Adrian Taylor for enabling me in my task of summarising this broad area of research literature.

\section{References}

1 WHO. Exercise for health. WHO/FIMS Committee on Physical Activity for Health. Bulletin of the World Health Organisation 1995; 73(2): 135-6.

2 US Department of Health and Human Services (PHS). Physical activity and health. A report of the Surgeon General (Executive Summary), 1996. Pittsburgh, PA: Superintendent of Documents.

3 Berlin JA, Colditz GA. A meta-analysis of physical activity in the prevention of coronary heart disease. Am. J. Epidemiol. 1990; 132: 612-28.

4 Powell KE, Thompson PD, Casperson CJ, Kendrick JS. Physical activity and the incidence of coronary heart disease. Ann. Rev. Pub. Health 1987; 8: 253-87.

5 Prentice AM, Jebb S. Obesity in Britain: Gluttony or sloth. $\mathrm{Br}$. Med.J. 1995; 311: 437-9.

6 Sports Council/HEA. Allied Dunbar National Fitness Survey: Main findings. 1992; London: Sports Council and HEA.

7 Powell KE, Blair S. The public health burdens of sedentary living habits: theoretical but realistic estimates. Medicine and Science in Sports and Exercise 1994; 26: 851-6.

8 Morris J. Exercise in the prevention of coronary heart disease: Today's best buy in public health. Medicine and Science in Sports \& Exercise 1994; 26: 807-14.

9 Prescott-Clarke P, Primatesta P, eds. Health Survey for England 1995: findings: A survey carried out on behalf of the Department of Health. 1997; London: The Stationary Office.

10 Kurtz Z. With health in mind. 1992; London: Action for Sick Children.

11 Rutter M, Smith DJ, eds. Psychosocial disorders in young people: Time trends and their causes. 1995; New York: John Wiley.

12 Weismann MM, Klerman GL. Depression: Current understanding and changing trends. Ann. Rev. Pub. Health 1992; 13: 319-39.

13 Paykel ES, Priest RG. Recognition and management of depression in general practice: A consensus statement. $\mathrm{Br}$. Med. J. 1992; 305: 1198-202.

14 Department of Health. NHS Executive. Burdens of disease: $a$ discussion document. 1996; London: Department of Health.

15 Morgan WP, Goldston SE, eds. Exercise and mental bealth 1987; Washington DC: Hemisphere.

16 Biddle SJH, Mutrie N. Psychology of physical activity and exercise. 1991; London: Springer-Verlag.

17 Leith LM. Foundations of exercise and mental health. 1994; Morgantown, WW: Fitness Information Technology.

18 Morgan WP, ed. Physical activity and mental health. 1997; Washington, DC: Taylor and Francis.

19 Van de Vliet P, Knapen J, Joos S, Van Coppenolle H, Pieters G. Psychomotor therapy for depressive psychiatric patients: Effects on the mood and self. Proceedings of the European
Congress on Sport Psychology, 1999, Prague, July. (part 2) pp. 271-3.

20 Beck AT, Ward CH, Mendelsohn M, Mock J, Erbaugh H. An inventory for measuring depression. Archives and General Psychiatry 1961; 4: 561-71.

21 American Psychiatric Association. Diagnostic and statistical manual of mental disorders ( $4^{\text {th }}$ edn) 1994; Washington, DC: APA.

22 Spitzer RL, Endicott J, Robins E. Research diagnostic criteria. Archives of General Psychiatry 1978; 35: 773-82.

23 Camacho TC, Roberts RE, Lazarus NB, Kaplan GA, Cohen RD. Physical activity and depression. Evidence from the Alameda County Study. Am.J. Epidemiol. 1991; 134: 220-31.

24 Paffenbarger RS, Lee I-M, Leung R. Physical activity and personal characteristics associated with depression and suicide in American college men. Acta Psychiatrica Scandinavia 1994; 89 (S377): 16-22.

25 Farmer ME, Locke BZ, Moscicki EK, Dannenberg AL, Larson DB, Radloff LS. Physical activity and depressive symptoms: The NHANES I Epidemiological Follow-Up Study. Am. J. Epidemiol. 1988; 128: 1340-51.

26 Mutrie N. The relationship between physical activity and clinically defined depression. In: Biddle SJH, Fox KR, Boutcher SH (eds) Physical activity and psychological well-being. London: Routledge (in press).

27 Craft LL, Landers DM. The effect of exercise on clinical depression and depression resulting from mental illness: A meta analysis. J. Sport Exercise Psychol. 1998; 20(4): 339-57.

28 Calfas KJ, Taylor WC. Effects of physical activity on psychological variables in adolescents. Pediatric Exercise Sci. 1994; 6(4): 406-23.

29 McDonald DG, Hodgdon JA. Psychological effects of aerobic fitness training, 1991; London: Springer-Verlag.

30 Petruzzello SJ, Landers DM, Hatfield BD, Kubitz KA, Salazar W. A meta-analysis on the anxiety-reducing effects of acute and chronic exercise. Outcomes and mechanisms. Sports Medicine 1991; 11(3): 143-82.

31 Taylor A. Physical activity stress and anxiety: A review. In: Biddle SJH, Fox KR, Boutcher SH (eds) Physical activity and psychological well-being. London: Routledge (in press).

32 Stephens T. Physical activity and mental health in the United States and Canada: Evidence from four population surveys. Preventive Medicine 1988; 17: 35-47.

33 Biddle SJH. Emotion, mood and physical activity. In: Biddle $\mathrm{SJH}$, Fox KR, Boutcher SH (eds) Physical activity and psychological well-being. London: Routledge (in press).

34 FOx KR. The effects of exercise on self-perceptions and selfesteem. In: Biddle SJH, Fox KR, Boutcher SH (eds) Physical activity and psychological well-being. London: Routledge (in press).

35 Spence JC, Poon P. The effect of physical activity on selfconcept: A meta-analysis. Alberta Centre for Well-Being. Research Update 1997; 4(3): 4.

36 Fox KR. The physical self and processes in self-esteem development. In: Fox KR, ed. The physical self: From motivation to well-being Champaign, IL: Human Kinetics. 1997; 111-40.

37 Sonstroem RJ, Potts SA. Life adjustment correlates of physical self-concepts. Med. Sci. Sports Exercise 1996; 28: 619-25.

38 Boutcher SH. Cognitive performance, fitness and ageing. In: Biddle SJH, Fox KR, Boutcher SH (eds) Physical activity and psychological well-being. London: Routledge (in press).

39 Etnier JL, Salazar W, Landers DM, Petruzzello SJ, Han M, Nowell P. The influence of physical fitness and exercise upon cognitive functioning: A meta-analysis. J. Sport Exercise Psychol. 1997; 19: 249-74.

40 National Commission on Sleep Disorders Research. Wake up America: A national sleep alert. Executive summary and executive report. $1993 ; 1-76$. 
41 Youngstedt SD, O'Connor PJ, Dishman RK. The effects of acute exercise on sleep: A quantitative synthesis. Sleep 1997; 20(3): 203-14.

42 Szabo A. Physical activity and psychological dysfunction. In: Biddle SJH, Fox KR, Boutcher SH (eds) Physical activity and psychological well-being. London: Routledge (in press).

43 Davis C. Body image, exercise and eating behaviours. In: Fox KR ed. The physical self: From motivation to well-being Champaign, IL: Human Kinetics. 1997: 143-74.

44 Hoffman P. The endorphin hypothesis. In: Morgan WP ed. Physical activity and mental health. Washington DC: Taylor \& Francis 1997: 163-78.

45 Dishman RK. The norepinephrine hypothesis. In: Morgan WP ed. Physical activity and mental health Washington DC: Taylor \& Francis 1997: 199-212.

46 Chaouloff F. The serotonin hypothesis. In: Morgan WP ed. Physical activity and mental health Washington DC: Taylor \& Francis. 1997: 179-98.

47 Koltyn KF. The thermogenic hypothesis. In: Morgan WP ed. Physical activity and mental health. Washington DC: Taylor \& Francis. 1997: 213-26.
48 McAuley E, Mihalko SL. Measuring exercise-related selfefficacy. In: Duda JL ed. Advances in sport and exercise psychology measurement Morgantown, WV: Fitness Information Technology. 1998: 371-92.

49 Harter S. Causes, correlates, and the functional role of global self-worth: A lifespan perspective. In: Sternberg RJ and Kolligian J. eds Competence considered. New York: VailBallou. 1990: 67-97.

50 Epling WF, Pierce WD. Activity-based anorexia in rats as a function of opportunity to run on an activity wheel. Nutrition and Behavior 1984; 2: 37-49.

51 Davis C, Kaptein S, Kaplan AS, Olmsted MP, Woodside DB. Obsessionality in anorexia nervosa: The moderating influence of exercise. Psychosomatic Medicine 1998; 60: 192-7.

52 Blundell JE, King NA. Effects of exercise on appetite control: loose coupling between energy expenditure and energy intake. Int. J. Obesity 1998; 22 (Suppl 2): S22-9.

53 Biddle SJH, Fox KR. Motivation for physical activity and weight management. Int. J. Obesity 1998; 22 (Suppl 2): S3947. 